\title{
Secondary breast cancer after Hodgkin lymphoma: a case report and literature review
}

\author{
Joaira Bakkach ${ }^{1}$, Mohamed Mansouri ${ }^{1,2}$, Ali Loudiyi ${ }^{2}$, Naima Ghailani Nourouti ${ }^{1}$, Amina Barakat ${ }^{1}$ and Mohcine Bennani Mechita ${ }^{1}$ \\ 'Biomedical Genomics and Oncogenetics Research Laboratory, Faculty of Sciences and Techniques of Tangier, University Abdelmalek Essaâdi, \\ Tangier 416, Morocco \\ ${ }^{2}$ Oncology Clinic AL AMAL of Tangier, 90 060, Morocco
}

Correspondence to: Joaira Bakkach. Email: joairaa@gmail.com

\begin{abstract}
The occurrence of secondary breast cancers in women previously exposed to chest irradiation for Hodgkin lymphoma (HL) is considered as a major issue for the quality of life of these long-term survivors as well as a challenge for clinical management. This study reports a case of a woman treated for $\mathrm{HL}$ at the age of 24 years, who developed breast cancer after an interval of 20 years. This case highlights once again the importance of awareness among HL survivors about their increased breast cancer risk and re-launches the debate about the efficacy of adoption of breast screening guidelines.
\end{abstract}

Keywords: Hodgkin lymphoma, secondary breast cancer, radiotherapy, cancer survivorship

ecancer 2018, 12:810 https://doi.org/10.3332/ecancer.2018.810

Copyright: (c) the authors; licensee ecancermedicalscience. This is an Open Access article distributed under the terms of the Creative Commons Attribution License (http://creativecommons.org/licenses/by/3.0), which permits unrestricted use, distribution, and reproduction in any medium, provided the original work is properly cited. 


\section{Background}

Patients treated for Hodgkin lymphoma $(\mathrm{HL})$ are faced with several late complications including the occurrence of secondary cancers, which is still a challenging issue in clinical oncology [1]. Breast cancer is the most common solid tumour in female survivors with an estimated relative risk of 8.23 compared to the general population [2]. Based on this increased risk, several international recommendations have been established for breast surveillance [3-5], but published data on surveillance practices raise concerns [6-8]. Up to 87\% of HL survivors do not join adequate breast screening programmes [7], and almost half of them seem to be unaware of their increased risk [6].

This study presents a case report of a woman, who was treated for HL at the age of 24 years and developed breast cancer after an interval of 20 years. We also critically discuss current preventive strategies, surveillance, and treatment modalities in secondary breast cancers with a focus on current guidelines.

\section{Case report}

A 44-year-old premenopausal woman without family history of breast cancer was diagnosed with ulcerous-budding bleeding breast tumour at the Oncology Clinic AL AMAL of Tangier (Morocco) with an 18-month history. On clinical examination, she was found to have a bleeding ulcerous mass in the upper outer quadrant of the right breast without axillary adenopathy (T4dNOMx).

She had been diagnosed with HL Classical Nodular Sclerosis subtype at the age of 24 years (1998). There was no evidence of bone marrow involvement on bone marrow biopsy. The disease was staged III according to Ann Arbor classification. The patient was treated by three cycles of MOPP chemotherapy (mechlorethamine, vincristine, procarbazine and prednisone), followed by 36Gy supra and infradiaphragmatic radiotherapy with up to $50 \mathrm{~Gy}$ localised boost on the left latero-cervical chain.

Infiltrating ductal carcinoma of the right breast, grade 2 was confirmed by biopsy. Estrogen receptors were positive in $70 \%$ of tumour cells, progesterone in $60 \%$ and Human Epidermal growth factor Receptor 2 (HER2) was scored 1+. Chest-abdomen-pelvis computed tomography revealed totally or partially calcified or hypodense masses, and nodules within mediastinum, right hilum and abdomen that were attributed to her previous HL. No bone metastases were found on the bone scan.

The patient was offered eight cycles of neoadjuvant chemotherapy based on adriamycin/cyclophosphamide and Taxotere (4 AC60 and 4 docetaxel), with partial clinical response $(70 \%)$. She subsequently underwent right mastectomy with level II axillary lymph node dissection. Histopathology examination showed a residual tumour classified pT3N2M0, grade 2. Following mastectomy, she received chest wall radiotherapy with a total dose of $50 \mathrm{~Gy}$ given at $2 \mathrm{~Gy}$ per fraction in 25 sessions over 5 weeks. Lymph nodes were not irradiated given her prior history of radiotherapy for HL. Radiation therapy was performed without significant complications, except a moderate radiodermatitis (grade 2) for which a local treatment was given. The patient received hormone therapy with Tamoxifen.

In April 2015, she presented with a skin nodule within the mastectomy scar. Cutaneous excision revealed locally recurrent infiltrating ductal carcinoma, grade 2, without endo-vascular emboli. In January 2017, she re-presented with an indurated left axilla associated with small satellite lymphadenopathy. Biopsy of the induration indicated fibrous mastosis without signs of malignancy. Similarly, axillary lymph node biopsy showed no suspicious malignant cells.

\section{Discussion}

It is well established that HL survivors have an increased risk of developing breast cancer when compared to the general population [1, 2, 9-14]. The cumulative risk has been shown to be almost similar to that of BRCA1 mutation carriers and to be 3-fold higher as compared with BRCA2 carriers (35\% versus $31 \%$ and $10 \%$, respectively) [9]. Importantly, this risk seems to start increasing $5-9$ years following the treatment, reaches a peak after 15-19 years [2] and it persists even up to 40 years of follow-up [1, 12]. 
The risk of secondary breast cancer occurrence was shown to be most influenced by age at treatment and importantly time since treatment and dose ( $>40 \mathrm{~Gy}$ yielding greatest risk) [2]. This risk seems to be higher among women exposed early to irradiation with the highest relative risk of 68.7 at 15 years or younger, and to be not significant among women diagnosed after the age of 40 [2] or 50 years [10]. Of note, our patient was diagnosed and treated at the age of 24 years (1998).

Treatment type, most notably radiotherapy with extensive field has been shown as an important risk factor for developing second breast cancer. In a study by De Bruin et al. [13], mantle irradiation has been associated with 2.7-fold increased relative risk compared to mediastinal irradiation alone. Furthermore, combination of any chemotherapy appears to promote the radiotherapy effect, and this was even more pronounced if chemotherapy was based on alkylating agents such as mechlorethamine and procarbazine [2]. However, other studies have demonstrated that additional alkylating chemotherapy is associated with reduced risk, and this was attributed to the protector effect of early menopause induced by this gonadotoxic chemotherapy [11-13]. In a cohort study published by Swerdlow et al. [12], individualised risk estimations have been provided according to several factors. For instance, a patient, who has been treated 20 years previously with $\geq 40 \mathrm{~Gy}$ mantle radiotherapy combined to alkylating chemotherapy at the age of 20-35 years, had a cumulative risk of 10.6 in the next 10 years. Our patient received MOPP chemotherapy followed by supra and infradiaphragmatic radiotherapies with a total cumulated dose of 86Gy, consistent with the guidelines available in the era when she was treated (1998).

Secondary breast cancers following HL generally occur at least 20 years younger than that of sporadic cancers with a median age of approximately 40 years [11, 14, 15]. Our patient was 42 years old at diagnosis.

These tumours were reported to be often bilateral [15], occur more frequently in the margins of radiotherapy field [2, 14], and to be more likely located on external quadrants [10].

Cardiac irradiation and anthracycline-based chemotherapy received during treatment for $\mathrm{HL}$ increase the risk of cardiac complications with an estimated 20 -year cumulative incidence rate of $16 \%$ [16].

This increased risk of cardiotoxicity may unfortunately limit the therapeutic options for women, who develop secondary breast cancer. Thus, several important clinical questions are raised. For instance, is there a place for conservative therapy for breast cancer patients with tumours in the left breast, or should they be treated systematically by mastectomy in order to avoid radiotherapy that may increase their cardiotoxicity risk? Is there a place for chest wall radiotherapy post mastectomy for the poor-prognosis breast cancer group with tumours in the left breast? Another raised issue is related to HER2-positive breast cancers - what will be the appropriate duration for Trastuzumab in this setting in light of the increased risk of cardiotoxicity with previous anthracycline and radiotherapy treatment? As these questions seem to be unresolved in randomised trials (including $\mathrm{HL}$ patients), individualised decision making by a multidisciplinary team remains the best option for second breast cancer management.

Mastectomy has long been described as the preferred surgical option in order to avoid further sequelae related to re-irradiation after conserving surgery, such as cardiotoxicity as well as tissue necrosis, which could develop many years thereafter [14]. However, the role of conservative therapy has been re-evaluated in some studies that have reported satisfying locoregional control and acceptable cosmetic results, suggesting that it could be a possible option for these survivors [17-30] (Table 1). Interestingly, despite the limited number of recruited patients and the low evidence level, some of these reports have shed light on promising radiotherapy techniques that could reduce mastectomy numbers including the intraoperative radiotherapy with electrons (ELIOT), partial breast irradiation (PBI) by brachytherapy or three-dimensional (3D) conformal techniques. There is a great need for prospective studies to validate these therapeutic options in HL survivors, and to determine the groups of patients who could benefit from it.

A Phase II trial conducted by the Radiation Therapy Oncology Group is underway in order to evaluate adverse events after 3D conformal $\mathrm{PBI}$ in locally recurrent breast cancer patients treated by repeat breast conserving surgery (NCT01082211) [31]. As clinical trials involving survivors with secondary breast cancer after HL are lacking, extrapolation from such studies could be important and may expand the treatment options for this group of patients.

Our patient underwent mastectomy due to the advanced stage of her disease (T4dNOMO). Based on the radiation oncologists' view and considering her good skin status, she was offered subsequent radiotherapy in the right chest wall, but without lymph nodes irradiation because of her prior irradiation for $\mathrm{HL}$. 
Table 1. Published studies reporting conservative treatment among breast cancer patients previously treated for HL.

\begin{tabular}{|c|c|c|c|c|c|c|}
\hline Study & $\begin{array}{l}\text { Re-irradiation } \\
\text { technique }\end{array}$ & Dose in Gy & $\begin{array}{c}\text { Median follow-up } \\
\text { in mo } \\
\text { (range) }\end{array}$ & Toxicity events & $\begin{array}{l}\text { Locoregional } \\
\text { recurrence rate } \\
(\%)\end{array}$ & Cosmetic result \\
\hline Wolden et al. [14] n = 2 & $\begin{array}{l}\text { WBRT with } \\
\text { tangential fields }\end{array}$ & $45.6,46$ & $72,168^{a}$ & $\begin{array}{l}\text { Severe soft tissue } \\
\text { necrosis }\end{array}$ & 0 & NA \\
\hline Aref et al. [22] n = 2 & WBRT & 46,48 & $27,32^{\mathrm{a}}$ & No & 0 & Good to excellent \\
\hline Deutsch et al. [18] n = 11 & WBRT & $50-51$ & $46(1-74)$ & No & 0 & Good to excellent \\
\hline Cutuli et al. [23] n = 32 & WBRT & NA & $40(10-192)$ & No & 12.5 & NA \\
\hline Intra et al. [17] n = 6 & ELIOT & 17,21 & $30.8^{\mathrm{b}}(17-53)$ & No & 8 & Very good \\
\hline Sana et al. [24] n = 33 & EBRT, ELIOT & NA & $41(1-101)$ & NA & $<5^{d}$ & NA \\
\hline El-Din et al. [19] n = 1 & 3D conformal PBI & 50 & $27^{\mathrm{a}}$ & Moderate erythema & 0 & Excellent \\
\hline Chadha et al. [25] n = 5 & PBB & 45 & $(5-67)$ & No & 0 & Excellent \\
\hline Nguyen et al. [21] n = 2 & WBRT & 45,50 & $43^{c}(6-127)$ & $\mathrm{G} 1^{\mathrm{c}}$ & 0 & NA \\
\hline Intra et al. $.^{c}[26] n=35$ & ELIOT & $17,18,21$ & $52(6-132)$ & $\begin{array}{l}\text { G1, G2 subcutaneous } \\
\text { tissue }\end{array}$ & 9 & Very good \\
\hline Cutuli et al. [27] n = 56 & WBRT & NA & 50 & No & 13.7 & NA \\
\hline Haberer et al. [20] n = 30 & WBRT & $39.6-52.2$ & $84(4.8-384)$ & NA & $7^{d}$ & NA \\
\hline Chadha et al. [28] n = 8 & PBB & $45^{e, f}$ & $73^{f}$ & $\begin{array}{l}\text { G1 skin pigmentation + } \\
\text { G3 fibrosis }\end{array}$ & $1^{\mathrm{d}}$ & Excellent \\
\hline Terenziani et al. [29] n = 29 & NA & $28-60$ & 99 & No & NA & NA \\
\hline Burt et al. [30] n = 46 & NA & NA & $57(0-299)$ & NA & NA & NA \\
\hline
\end{tabular}

a: Follow-up in months; ${ }^{\text {: }}$ : Average follow-up; c: Data for HL+NHL; d: Number of locoregional recurrences; e: Median dose; f: Data for patients with prior $\mathrm{HL}+$ prior history of breast cancer.

Abbreviations: EBRT: External beam radiation therapy; ELIOT: Intraoperative radiotherapy with electrons; G: Grade; NA: Not available; PBB: Partial breast brachytherapy; PBI: Partial breast irradiation; WBRT: Whole breast radiotherapy.

Over the years, with the accumulating evidence supporting the increased risk of late complications related to older therapy modalities, the latter have been adapted and evolved significantly. Extended-field irradiation (mantle-type, lumbosplenic bar or inverted-Y), which was the standard of care for many decades has been replaced by involved field radiotherapy (IFRT). Based on this evolutionary concept of IFRT, several techniques have been developed later such as involved node radiotherapy and involved site radiotherapy [32]. The higher doses routinely administered in the past were also de-escalated from more than 40Gy to 30Gy or 20Gy [32] and with the advent of computed tomography, conformational imaging and now sophisticated dynamic imaging techniques used in planning, delivery of treatment is much more precise.

This de-escalation in fields and doses has been shown to permit greater protection of healthy breast tissue from the exposure to the radiation effect. Additionally, some evidence has suggested that this has been associated with significantly reduced breast cancer risk, although the risk still persists higher compared to general population [1, 9, 12, 13, 33]. Using a dosimetric risk modelling approach, Koh et al. [33] have demonstrated that the transition from 35Gy mantle radiotherapy to 35Gy IFRT has reduced the excess risk of breast cancer by $65 \%$ and the same risk is even more reduced by $40 \%$ when moving to 20 Gy IFRT.

Prevention and surveillance modalities are also key in addressing the increased risk of secondary breast cancer. The evidence for the efficacy of most prevention and screening interventions however is generally extrapolated from other high-risk groups particularly BRCA1 or BRCA2 mutation carriers and has not specifically been measured in pretreated patient populations.

The role of prophylactic bilateral mastectomy in prevention is yet to be defined in this group. A randomised placebo-controlled phase IIB trial is underway to assess whether low-dose Tamoxifen for 2 years may reduce the risk of radiation-induced breast cancer in patients who have 
received thoracic irradiation (NCT01196936) [34]. However, this chemoprevention will not be helpful in preventing hormone receptor-negative breast cancers [35], which are the most frequent histological subtype that develop in HL survivors according to some reports [2, 36].

Generally, the primary aim of breast cancer screening is to reduce breast cancer mortality, and this should be assessed in randomised controlled trials. However, no such study has been unfortunately conducted among HL survivors, and the only data available so far that show the impact on mortality for this group of patients are provided by a mathematical simulation study published by Hodgson et al. [37]. This model has predicted for the first time that early screening (at 25 years) will reduce breast cancer mortality among HL survivors treated with mediastinal radiotherapy. More specifically, early detection with magnetic resonance imaging (MRI) over a 24-year period will prevent 13.92 to 14.35 deaths from breast cancer.

Data from the UK screening program has shown that most second breast cancers were detected at a pre-invasive or early stage and were without lymph node involvement [5], from which it would be reasonable to extrapolate that screening will yield clinical benefit for these patients but this has yet to be confirmed in terms of mortality outcomes.

The International Late Effects of Childhood Cancer Guideline Harmonization Group has proposed annual breast surveillance for HL survivors, who have received $\geq 20 \mathrm{~Gy}$ thoracic radiotherapy before the age of 30 years [3]. Surveillance should start at 25 or at least 8 years after irradiation for at least up to 50 years, and should include mammography, MRI or both [3]. The ideal imaging modality is still controversial. Mammography appears to be able to detect most secondary breast cancers even in young women with dense breasts [6, 14]. Nonetheless, it exposes the body to additional radiation doses. Interestingly, combining breast MRI to mammography has showed increased sensitivity from $68 \%$ to $94 \%$ [38], but this is at the expense of higher costs, as well as further stress linked to the increased number of false-positive results, leading to unnecessary exams and biopsies.

Despite the international efforts to establish surveillance recommendations for HL survivors, a great proportion including our patient did not even know about their increased risk of developing secondary breast cancer and had never undergone neither self-breast exam nor mammography [6-8]. Thus, better awareness of clinicians about these risks can help with communication to these women about secondary breast cancer risk and should contribute to improved risk management.

\section{Conclusion}

The de-escalation of $\mathrm{HL}$ treatments that has occurred over the years, with the limitation and more precise delivery of irradiated disease fields and the reduction of radiation doses, has paved the way for what we hope will be a significantly reduced risk of second breast cancer in this group of patients. Yet, there is still much to be done in raising awareness among survivors for their increased breast cancer risk.

The need to determine what constitutes adequate breast screening in these patients is also important, but studies with a high evidence level are needed to better evaluate benefits and limitations.

Finally, in view of the increased risk of cardiotoxicity and in the absence of a consensus for these survivors, individualised and concerted management remains the best option for the treatment of secondary breast cancer after $\mathrm{HL}$.

\section{Abbreviations}

EBRT: external beam radiation therapy; ELIOT: intraoperative radiotherapy with electrons; G: grade; HER2: human epidermal growth factor receptor 2; HL: Hodgkin lymphoma; IFRT: involved field radiotherapy; MRI: magnetic resonance imaging; mSBR: Scarf-Bloom-Richardson classification modified by Elston and Elliss; NA: not available; PBB: partial breast brachytherapy; PBI: partial breast irradiation; WBRT: whole breast radiotherapy.

\section{Competing interests}

The authors declare that they have no competing interests. 


\section{Authors' contributions}

JB designed the study, performed the literature review, drafted the manuscript and carried out the collection and assembly of data. MM analysed and interpreted the patient data regarding her oncological features and contributed to the revision of the manuscript. AL is responsible for the clinical management. All authors read and approved the final manuscript.

\section{Acknowledgments}

The authors acknowledge the contribution of Dr Khalid El Bairi (Independent Research Team in Cancer Biology and Bioactive Compounds, Mohamed 1st University, Oujda, Morocco) in reviewing the manuscript.

\section{References}

1. Schaapveld M, Aleman BM, and Van Eggermond AM, et al (2015) Second cancer risk up to 40 years after treatment for Hodgkin's lymphoma N Engl J Med 373 2499-2511 https://doi.org/10.1056/NEJMoa1505949 PMID: 26699166

2. Ibrahim EM, Abouelkhair KM, and Kazkaz GA, et al (2012) Risk of second breast cancer in female Hodgkin's lymphoma survivors: a meta-analysis BMC Cancer 12197 https://doi.org/10.1186/1471-2407-12-197

3. Mulder RL, Kremer LC, and Hudson MM, et al (2013) Recommendations for breast cancer surveillance for female survivors of childhood, adolescent, and young adult cancer given chest radiation: a report from the International Late Effects of Childhood Cancer Guideline Harmonization Group Lancet Oncol 14 e621-e629 https://doi.org/10.1016/S1470-2045(13)70303-6 PMID: 24275135 PMCID: 4257601

4. Radford J, Howell S, and O'Hara C, et al (2013) The breast cancer after radiotherapy dataset (BARD): an initiative to improve screening for breast cancer in a high risk cohort of female Hodgkin lymphoma survivors in England Haematologica 98 s2

5. Howell SJ, Searle C, and Goode V, et al (2009) The UK national breast cancer screening programme for survivors of Hodgkin lymphoma detects breast cancer at an early stage Br J Cancer 101 582-588 https://doi.org/10.1038/sj.bjc.6605215 PMID: 19672261 PMCID: 2736813

6. Diller L, Nancarrow CM, and Shaffer K, et al (2002) Breast cancer screening in women previously treated for Hodgkin's disease: a prospective cohort study J Clin Oncol 20 2085-2091 https://doi.org/10.1200/JC0.2002.08.031 PMID: 11956269

7. Hodgson DC, Grunfeld E, and Gunraj N, et al (2010) A population-based study of follow-up care for Hodgkin lymphoma survivors: opportunities to improve surveillance for relapse and late effects Cancer 116 3417-3425 https://doi.org/10.1002/cncr.25053 PMID: 20564062

8. Nathan PC, Ness KK, and Mahoney MC, et al (2010) Screening and surveillance for second malignant neoplasms in adult survivors of childhood cancer: a report from the childhood cancer survivor study Ann Intern Med 153 $442-451$ https://doi. org/10.7326/0003-4819-153-7-201010050-00007 PMID: 20921543 PMCID: $\underline{3084018}$

9. Moskowitz CS, Chou JF, and Wolden SL, et al (2014) Breast cancer after chest radiation therapy for childhood cancer J Clin Oncol 32 2217-2223 https://doi.org/10.1200/JCO.2013.54.4601 PMID: 24752044 PMCID: 4100937

10. Veit-Rubin N, Rapiti E, and Usel M, et al (2012) Risk, characteristics, and prognosis of breast cancer after Hodgkin's lymphoma Oncologist 17 783-791 https://doi.org/10.1634/theoncologist.2011-0451 PMCID: 3380877

11. Travis LB, Hill DA, and Dores GM, et al (2003) Breast cancer following radiotherapy and chemotherapy among young women with Hodgkin disease JAMA 290 465-475 https://doi.org/10.1001/jama.290.4.465 PMID: 12876089 
12. Swerdlow AJ, Cooke R, and Bates A, et al (2012) Breast cancer risk after supradiaphragmatic radiotherapy for Hodgkin's lymphoma in England and Wales: a national cohort study J Clin Oncol 30 2745-2752 https://doi.org/10.1200/JCO.2011.38.8835 PMID: $\underline{22734026}$

13. De Bruin ML, Sparidans J, and van't Veer MB, et al (2009) Breast cancer risk in female survivors of Hodgkin's lymphoma: lower risk after smaller radiation volumes J Clin Oncol 27 4239-4246 https://doi.org/10.1200/JCO.2008.19.9174 PMID: 19667275

14. Wolden SL, Hancock SL, and Carlson RW, et al (2000) Management of breast cancer after Hodgkin's disease J Clin Oncol 18 765-772 https://doi.org/10.1200/JCO.2000.18.4.765 PMID: 10673517

15. Elkin EB, Klem ML, and Gonzales AM, et al (2011) Characteristics and outcomes of breast cancer in women with and without a history of radiation for Hodgkin's lymphoma: a multi-institutional, matched cohort study J Clin Oncol 29 2466-2473 https://doi. org/10.1200/JCO.2010.32.4079 PMID: 21576642 PMCID: 3138631

16. Galper SL, James BY, and Mauch PM, et al (2011) Clinically significant cardiac disease in patients with Hodgkin lymphoma treated with mediastinal irradiation Blood 117 412-418 https://doi.org/10.1182/blood-2010-06-291328

17. Intra M, Gentilini O, and Veronesi P, et al (2005) A new option for early breast cancer patients previously irradiated for Hodgkin's disease: intraoperative radiotherapy with electrons (ELIOT) Breast Cancer Res 7 R828-R832 https://doi.org/10.1186/bcr1310

18. Deutsch M, Gerszten K, and Bloomer WD, et al (2001) Lumpectomy and breast irradiation for breast cancer arising after previous radiotherapy for Hodgkin's disease or lymphoma Am J Clin Oncol 24 33-34 https://doi.org/10.1097/00000421-200102000$\underline{00005}$ PMID: 11232946

19. Alm El-Din MA, Feng JK, and Taghian AG (2008) Lumpectomy and partial breast irradiation for early-stage breast cancer following mantle irradiation for Hodgkin's lymphoma Nat Clin Pract Oncol 5 426-429 https://doi.org/10.1038/ncponc1135 PMID: 18493230

20. Haberer S, Belin L, and Le Scodan R, et al (2012) Locoregional treatment for breast carcinoma after Hodgkin's lymphoma: the breast conservation option Int J Radiat Oncol 82 e145-e152 https://doi.org/10.1016/j.jirobp.2011.03.013

21. Nguyen SK and Dagnault A (2010) Breast-conserving therapy after previous irradiation for lymphoma Breast Cancer Res Treat 124 845-849 https://doi.org/10.1007/s10549-009-0421-2

22. Aref I and Cross $P$ (2000) Conservative surgery and radiation therapy for early stage breast cancer after previous mantle radiation for Hodgkin's disease Br J Radiol 73 905-906 https://doi.org/10.1259/bjr.73.872.11026871 PMID: 11026871

23. Cutuli B, Borel C, and Dhermain F, et al (2001) Breast cancer occurred after treatment for Hodgkin's disease: analysis of 133 cases Radiother Oncol 59 247-255 https://doi.org/10.1016/S0167-8140(01)00337-1 PMID: 11369065

24. Sanna G, Lorizzo K, and Rotmensz N, et al (2006) Breast cancer in Hodgkin's disease and non-Hodgkin's lymphoma survivors Ann Oncol 18 288-292 https://doi.org/10.1093/annonc/mdl399

25. Chadha M, Yoon H, and Feldman S, et al (2009) Partial breast brachytherapy as the primary treatment for breast cancer diagnosed after mantle radiation therapy for Hodgkin's disease Am J Clin Onco/ 32 132-136 https://doi.org/10.1097/COC.0b013e318180c880 PMID: 19307955

26. Intra M, Mattar D, and Sangalli C, et al (2011) Local therapy for breast cancer in malignant lymphoma survivors Breast 20 S99S103 https://doi.org/10.1016/S0960-9776(11)70304-6 PMID: 22015303

27. Cutuli B, Kanoun S, and De Lara CT, et al (2012) Breast cancer occurred after Hodgkin's disease: clinico-pathological features, treatments and outcome: analysis of 214 cases Crit Rev Oncol Hematol 81 29-37 https://doi.org/10.1016/j.critrevonc.2011.01.005

28. Chadha M, Boolbol SK, and Kirstein L, et al (2013) Patterns of relapse and risk for new malignancies following partial breast re-irradiation J Radiat Oncol 2 43-48 https://doi.org/10.1007/s13566-012-0073-4 
29. Terenziani M, Massimino M, and Magazzù D, et al (2015) Management of breast cancer after Hodgkin's lymphoma and paediatric cancer Eur J Cancer 51 1667-1674 https://doi.org/10.1016/j.ejca.2015.05.024 PMID: 26092639

30. Burt LM, Poppe M, and Kokeny KE, et al (2017) Comparison of treatment modalities for breast cancer arising in Hodgkin's lymphoma survivors J Radiat Oncol 6 65-72 https://doi.org/10.1007/s13566-016-0262-7

31. Radiation therapy in treating women with locally recurrent breast cancer previously treated with repeat breast-preserving surgery [https://clinicaltrials.gov/ct2/show/NCT01082211] Date accessed: 02/10/17

32. Specht L, Yahalom J, and Illidge T, et al (2014) Modern radiation therapy for Hodgkin lymphoma: field and dose guidelines from the International Lymphoma Radiation Oncology Group (ILROG) Int J Radiat Oncol 89 854-862 https://doi.org/10.1016/j. ïrobp.2013.05.005

33. Koh ES, Tran TH, and Heydarian M, et al (2007) A comparison of mantle versus involved-field radiotherapy for Hodgkin's lymphoma: reduction in normal tissue dose and second cancer risk Radiat Oncol 2 13 https://doi.org/10.1186/1748-717X-2-13

34. Low-dose Tamoxifen Citrate in reducing breast cancer risk in radiation-induced cancer survivors [https://clinicaltrials.gov/ct2/ show/NCT01196936] Date accessed: 30/07/17

35. Manzo V, Dirbas F, and Telli ML (2016) Breast cancer after Hodgkin lymphoma: the price of success Oncology (Williston Park) 30 1072-1073

36. Dores GM, Anderson WF, and Beane Freeman LE, et al (2010) Risk of breast cancer according to clinicopathologic features among long-term survivors of Hodgkin's lymphoma treated with radiotherapy Br J Cancer103 1081-1084 https://doi.org/10.1038/ sj.bjc.6605877 PMID: 20842115 PMCID: 2965878

37. Hodgson DC, Cotton C, and Crystal P, et al (2016) Impact of early breast cancer screening on mortality among young survivors of childhood Hodgkin's lymphoma J Natl Cancer Inst 108 djw010 https://doi.org/10.1093/jnci/djw010

38. $\mathrm{Ng} \mathrm{AK}$, Garber JE, and Diller LR, et al (2013) Prospective study of the efficacy of breast magnetic resonance imaging and mammographic screening in survivors of Hodgkin lymphoma J Clin Oncol 31 2282-2288 https://doi.org/10.1200/JCO.2012.46.5732 PMID: 23610104 